\title{
Martini Coarse-Grained Model of Hyaluronic Acid for the Structural Change of Its Gel in the Presence of Monovalent and Divalent Salts
}

\author{
Raj Kumar ${ }^{1,2}$, Young Kyu Lee ${ }^{1}$ and Yong Seok Jho ${ }^{1, *}$ \\ 1 Department of Physics and Research Institute of Natural Science, Gyeongsang National University (GNU), \\ 501 Jinju-daero, Jinju 52828, Korea; raj.kumar@juit.ac.in (R.K.); monsterplank@gmail.com (Y.K.L.) \\ 2 Department of Biotechnology and Bioinformatics, Jaypee University of Information Technology (JUIT), \\ Waknaghat, Solan 173234, India \\ * Correspondence: ysjho@gnu.ac.kr
}

Received: 25 May 2020; Accepted: 25 June 2020; Published: 29 June 2020

\begin{abstract}
Hyaluronic acid (HA) has a wide range of biomedical applications including the formation of hydrogels, microspheres, sponges, and films. The modeling of HA to understand its behavior and interaction with other biomolecules at the atomic level is of considerable interest. The atomistic representation of long HA polymers for the study of the macroscopic structural formation and its interactions with other polyelectrolytes is computationally demanding. To overcome this limitation, we developed a coarse grained (CG) model for HA adapting the Martini scheme. A very good agreement was observed between the CG model and all-atom simulations for both local (bonded interactions) and global properties (end-to-end distance, a radius of gyration, RMSD). Our CG model successfully demonstrated the formation of HA gel and its structural changes at high salt concentrations. We found that the main role of $\mathrm{CaCl}_{2}$ is screening the electrostatic repulsion between chains. HA gel did not collapse even at high $\mathrm{CaCl}_{2}$ concentrations, and the osmotic pressure decreased, which agrees well with the experimental results. This is a distinct property of HA from other proteins or polynucleic acids which ensures the validity of our CG model. Our HA CG model is compatible with other CG biomolecular models developed under the Martini scheme, which allows for large-scale simulations of various HA-based complex systems.
\end{abstract}

Keywords: hyaluronic acid; gel formation; coarse-grained simulation; Martini

\section{Introduction}

Hyaluronic acid (HA), also called hyaluronan, is a linear anionic polysaccharide comprised of repeating disaccharide units of D-glucuronic acid (GlcA) and N-acetyl-D-glucosamine (GlcNAc) linked by alternating $\beta(1-3)$ and $\beta(1-4)$ glucosidic bonds [1]. HA is widely distributed and found in several locations in the body such as skin, synovial fluid, eyes, and lungs. HA is a component of the extracellular matrix (ECM), and its primary functions are to trap water inside tissue cells, maintain the moisture of eyes, and keep the joints well lubricated. HA can be chemically modified through conjugation and crosslinking polymerization processes, and its properties have been customized for several biomedical applications [2]. An interesting property of HA is that it keeps a liquid form in the physiological $\mathrm{NaCl}$ conditions, even under high $\mathrm{CaCl}_{2}$ concentrations, which is unusual for other biological polyelectrolytes. Due to this property and the high biocompatibility of HA, it has been widely investigated for the development of hydrogel scaffolds for tissue engineering [3]. The use of HA-based dermal fillers reduces the depth of skin folds and is used for facial rejuvenation in the area of cosmetic surgery. Different polyelectrolyte polymers have been studied in complex coacervate formation with HA. HA complex coacervation has also been investigated with different proteins 
such as silk fibroin and lysozyme. HA coacervate has been used to isolate the target protein bovine serum albumin from a mixture of $\beta$-lactoglobulin based on its electrostatic charge properties [4]. HA complexation stabilized the nanoparticles used in various gene silencing and gene therapy strategies. Several other formulations of covalently bound or cross-linked HA molecules forming hydrogel networks have been used for controlled-release drug delivery applications. HA polymers have been investigated in several other biomedical applications including tissue engineering, dermal filler for skin rejuvenation, visco-supplementation for arthritis, wound healing, ocular treatment, dermatology, plastic surgery, drug delivery, the delivery of therapeutic and biological competent cells, vaccine delivery, and gene therapy. Polyelectrolyte gels are considered as a model to study swelling behavior in several theoretical and applied research studies including muscle contraction, protein transitions, and nerve excitation [5-7]. Chemically crosslinked polyelectrolyte gels have greater swelling capacities than non-ionic polymer gels, which are advantageous in biomedical applications. Polyelectrolyte gel swelling is the subject of numerous studies in polymer physics which demonstrate that minute changes in external conditions such as temperature, external electric field, solvent composition, and ionic strength can induce drastic changes in gel swelling properties [8]. Exposure to multivalent counterions causes the precipitation of polyelectrolytes, which poses a huge problem in bioengineering [9]. However, HA does not precipitate at high multivalent counterion concentrations, which distinguishes this molecule from other biopolymers such as DNA [10]. This study correlates the behavior of $\mathrm{HA}$ gel formation in varying concentrations of the monovalent salt $\mathrm{NaCl}$ and the divalent salt $\mathrm{CaCl}_{2}$ from simulations to actual laboratory experiments.

A complex coacervation process is the binding of two oppositely charged molecules facilitated mainly by electrostatic interaction, ultimately leading to a liquid-liquid phase separation and coacervate formation [11]. Additionally, hydrophobic-hydrophobic interactions, van der Waals intermolecular force, and intermolecular hydrogen bonding also play important roles in the mechanism of gel formation such as complex coacervation. Several molecular simulation approaches, such as Monte Carlo, Langevin dynamics, and molecular dynamics simulations, can be used to understand this polyelectrolyte complexation [12,13]. A computational demonstration of a complex process of coacervate gel formation may require simulation time scales that are too large to be studied in atomistic detail. However, lowering the level of polymer representation from all-atom to coarse-grained (CG) opens up new possibilities for studying polymer complexation and its phase separation behavior [14]. CG models based on the Martini scheme have been used for commonly used synthetic polymers such as polycarbonates, polystyrene, polyamide, polyethylene, and polypropylene [15-18]. Further, coarse-grained studies of the self-assembly mechanism of Pluronic or poloxamer triblock copolymers reproducing experimental micelle sizes and shapes have been reported $[19,20]$. Martini-based models have also been applied to understand the cross interactions of protein-polymer complex formations [21]. Several recent reports justify the application of Martini CG models to a variety of molecules, including calcein fluorescent dye [22], polyethylenimine [23], clay-polymer nanocomposites [24], poly(3,4-ethylenedioxythiophene) (PEDOT) [25], etc. Moreover, Martini-based CG models have been successfully applied to study supramolecular polymer assemblies such as benzene-1,3,5-tricarboxamide (BTA) [26,27] and peptide amphiphiles [28,29]. Additionally, Martini parameters have been developed for biopolymers and a wide variety of biomolecules such as membrane lipids, glycolipids, carbohydrates, proteins, DNA, and RNA [30]. Continuous efforts for developing transferable Martini models have been reported for several molecules, including polyethylene oxide (PEO) [31], polyethers [32], perfluorosulfonic acid polymer membranes [33], and methacrylate-based copolymers [34]. Martini models have also been successful in studies capturing the phase behavior and phase transitions of several polymer systems [35-41]. The above examples justify the wide acceptability of Martini CG models for various compounds and polymer systems. In this study, we developed and validated a CG model for HA based on the Martini scheme for modeling coarse-grained simulations, which will make it possible to perform greatly accelerated simulations for understanding events in HA polyelectrolyte complexation. We applied 
our model to studies on the structural change of HA gel in the presence of monovalent and divalent salts, the microscopic mechanism of which is not yet clearly understood. Our computational studies rendered the microscopic mechanism of the previous experiments. The HA CG model presented here will make it possible to perform greatly accelerated simulations for understanding events in HA polyelectrolyte complexation.

\section{Results and Discussion}

\subsection{Measures of CG Simulations}

Here, we develop the CG model of hyaluronic acid (HA) by the basic principles described in the Methods section. HA is a linear polymer made up of repeating disaccharide units of GlcA and GlcNAc connected by $\beta 1-3$ (HA1-3) and $\beta 1-4$ (HA1-4) linkages (Figure 1). Therefore, we modeled two different bonding monosaccharide units separately and incorporated the obtained parameters to model a larger molecule of HA containing eight monosaccharide units [42].

A

$\beta 1-3$ linkage<smiles>CC(=O)N[C@H]1COC(CO)[C@H](O)[C@H]1OC1=C(O)C(O)C(O)C(C(=O)[O-])O1</smiles>

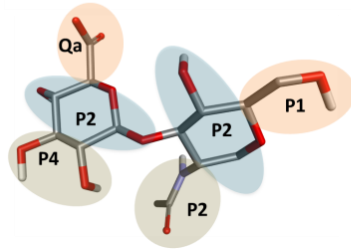

B $\beta 1-4$ linkage

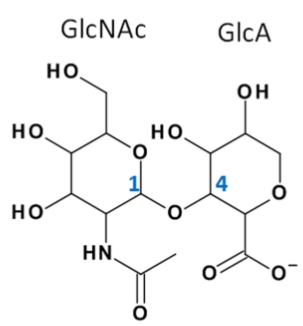

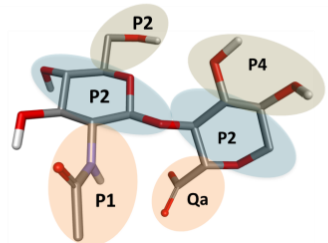

C
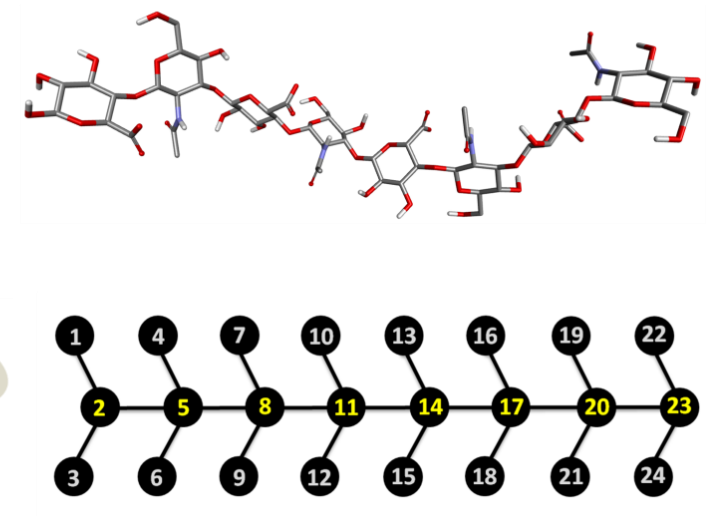

Figure 1. Chemical structures (top) and atomistic visualization (bottom) of HA1-3 and HA1-4 disaccharides (A,B). (C) Atomistic representation (top) of octasaccharide hyaluronic acid (HA) and its coarse-grained (CG) representation (bottom).

\subsubsection{Matching AA and CG Bonded Distributions}

Determining the parameters for bonded terms is an iterative process where initial values are first guessed, followed by tuning these values by trial and error. First, we obtained trajectories of all-atom (AA) simulations for all HA structures. All the AA trajectories were then converted to CG trajectories based on the CG mapping scheme described above. The probability distributions for the bonded terms could be calculated from these obtained CG trajectories which will be referred to as the AA distributions. The gmx distance program was used to calculate the bond distributions between averaged CG points obtained by averaging from AA simulations. The angles and dihedral distributions were calculated by using the gmx angle program. Secondly, a random guess for bonded terms was made to obtain initial parameters for running the CG simulations. The new probability distributions for bond lengths, angles, and dihedrals were calculated from the obtained CG trajectories, which are referred to as the CG distributions. The CG distributions were compared with the corresponding averaged AA distributions in order to obtain a good match. In case of a mismatch, CG parameters were continuously updated to reduce the distance between the two sets of distributions until a good match was obtained. 


\subsubsection{Global Properties}

Global properties of HA polymer such as the end-to-end distance $\left(R_{e}\right)$ and radius of gyration $\left(R_{g}\right)$ were calculated for both AA and CG distributions (Equation (1)). The $R_{e}$ and $R_{g}$ were calculated by using the gmx polystat program which plots the static properties of polymers as a function of time and prints the average value.

$$
R_{g}=\left(\frac{\sum_{i}\left\|r_{i}\right\|^{2} m_{i}}{\sum_{i} m_{i}}\right)
$$

where $i$ and $r_{i}$ are the position of atom $i$ with respect to the center of mass of the molecule and $m_{i}$ is the mass of the atom. The root mean square deviation (RMSD) values were calculated using the gmx rms program (Equation (2)). The RMSD of certain atoms in a molecule with respect to a reference structure can be calculated by least-square fitting the structure to the reference structure $\left(t_{2}=0\right)$.

$$
\operatorname{RMSD}\left(t_{1}, t_{2}\right)=\left[\frac{1}{M} \sum_{i=1}^{N} m_{i}\left\|r_{i}\left(t_{1}\right)-r_{i}\left(t_{2}\right)\right\|^{2}\right]^{\frac{1}{2}}
$$

where $M=\sum_{i=1}^{N} m_{i}$, and $r_{\mathbf{i}}(t)$ is the position of atom $i$ at time $t$.

\subsection{Comparison between $A A$ and $C G$ Simulations}

\subsubsection{Bonded Parameters}

There were a total of 26 different bonded distributions for two dimers of GlcA and GlcNAc linked through HA1-3 and HA1-4 linkages (Figures 2-4). The values of equilibrium bond lengths, bond angles, and dihedral angles with their respective force constants $\left(K_{a}, K_{b}\right.$, and $\left.K_{d}\right)$ were adjusted so that a good agreement between AA and CG bonded distributions was achieved. The details of the bonded parameters for all bond lengths, bond angles, and dihedral angles present in the HA structures obtained after many trial-and-error iterations are given in Figures 2-4. The bond lengths obtained from AA and CG simulations are computed and compared in Figure 2. In the case of HA1-3, the time-averaged CG bond lengths overlapped well and showed similar lengths as AA bonds. The average bond lengths between 1-2, 2-3, 2-5, 4-5, and 5-6 were $2.67 \AA, 2.23 \AA, 5.04 \AA, 3.56 \AA$, and 2.62 $\AA$, respectively. The longest bond was observed between beads $2-5$, which represents the glycosidic linkage between two monosaccharide subunits. The average bond lengths in the HA1-4 dimer showed very minute differences in AA and CG distributions (Figure 2B). The maximum difference observed between two distributions was $0.4 \AA$ between beads 1 and 2. However, the CG distributions still matched the averaged AA distributions reasonably well, and a similar degree of discrepancy has been accepted and reported elsewhere [23]. Interestingly, there was a noticeably large difference in average bond lengths of glycosidic bonds (2-5) in HA1-3 (5.04 $\AA$ ) and HA1-4 (6.21 $\AA$ ) dimers, showing the importance of CG mapping two HA dimers separately. 

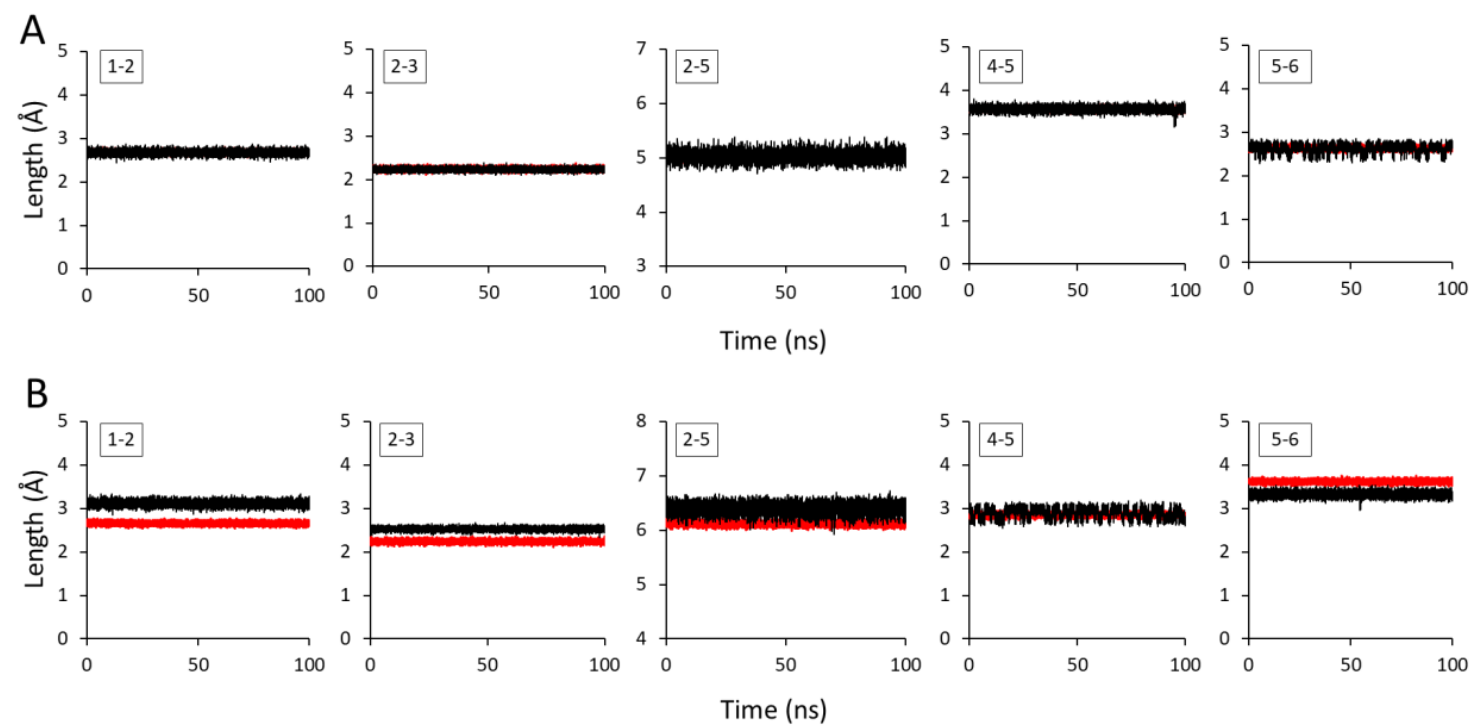

Figure 2. Bond lengths of all-atom (AA) and coarse-grained (CG) representations for (A) HA1-3 and

(B) HA1-4. Black and red lines indicate AA and CG representations, respectively.

Several adjustments of the equilibrium angle $\left(\theta_{\mathrm{eq}}\right.$ in Equation (8)) and force constant $\left(\mathrm{K}_{\mathrm{a}}\right)$ were made which resulted in a perfect match between AA and CG angle distributions of both HA1-3 and HA1-4 dimers (Figure 3). A slight increase in the $K_{a}$ values generally led to a sharpening of the probability distribution peaks, as observed in angle 2-5-4 in HA1-3. A relatively large $K_{a}(600)$ was used for angle 3-2-5 of the HA1-3 dimer, which is the angle between a P4 bead and two of main chain (MC) P2 beads. An acceptable discrepancy was observed in angle 3-2-5 of the HA1-4 dimer where the AA bond angle was $85^{\circ}$ and the CG angle distribution was observed is at around $80^{\circ}$.
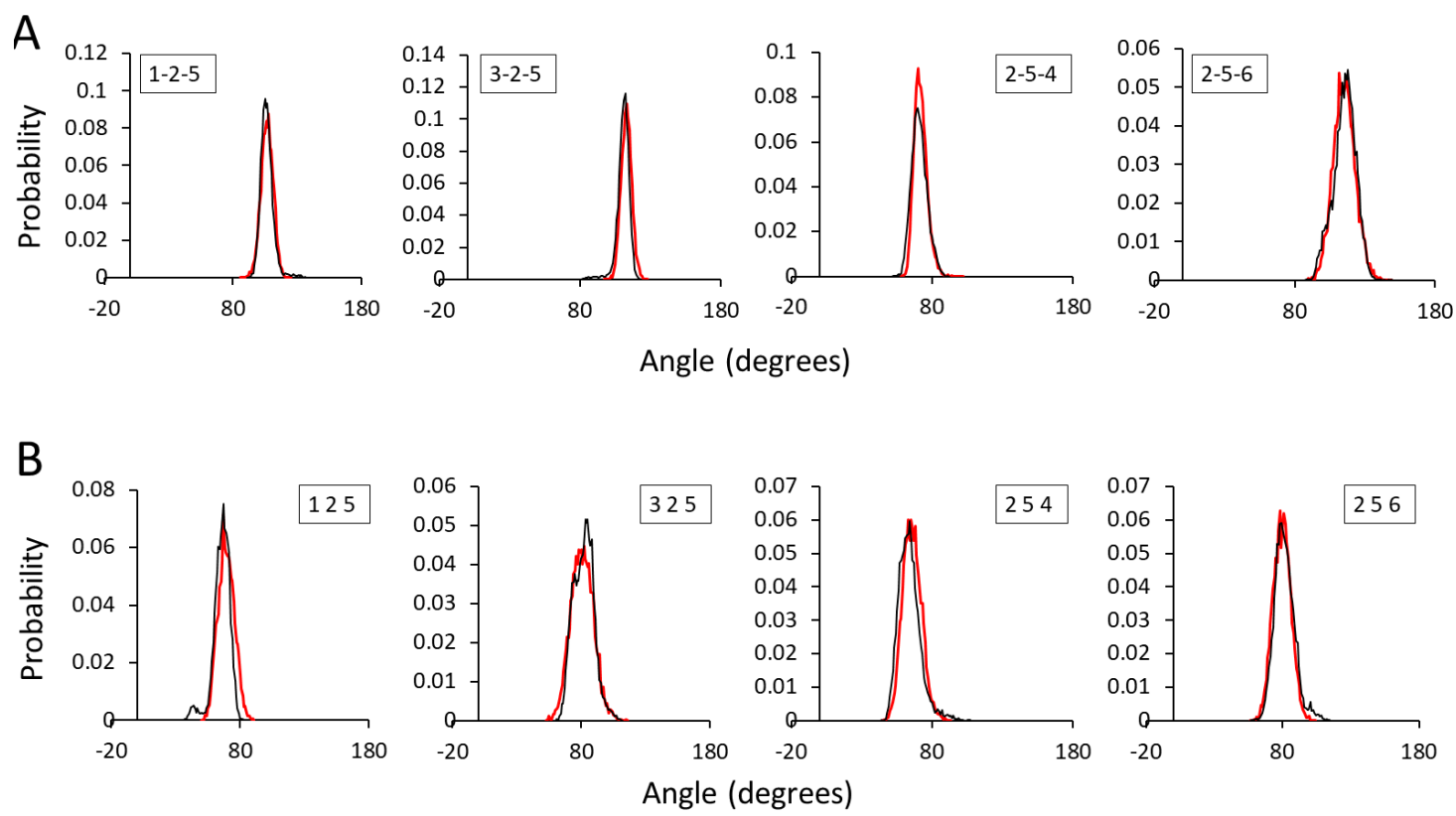

Figure 3. Angle distributions of AA and CG representations for (A) HA1-3 and (B) HA1-4. Black and red lines indicate AA and CG representations, respectively.

Comparatively larger fluctuations were observed between AA and CG dihedral angle distributions for the HA1-3 and HA1-4 dimers (Figure 4). Practically, the dihedral distributions are relatively more difficult to match among other bonded distributions, and large discrepancies have previously been 
reported [43]. As four beads are involved in dihedral bond formation, the most probable reason behind large fluctuations is steric hindrance among CG beads. The dihedral angles showed good agreement between AA and CG distributions in both HA1-3 and HA1-4 dimers. Several CG distributions have properly captured the location of the peaks of the distributions while only a few underestimated or overestimated certain peak heights and angles. Nonetheless, the CG distributions still match the averaged AA distributions reasonably well, and a similar degree of discrepancy has previously been reported and accepted $[43,44]$.
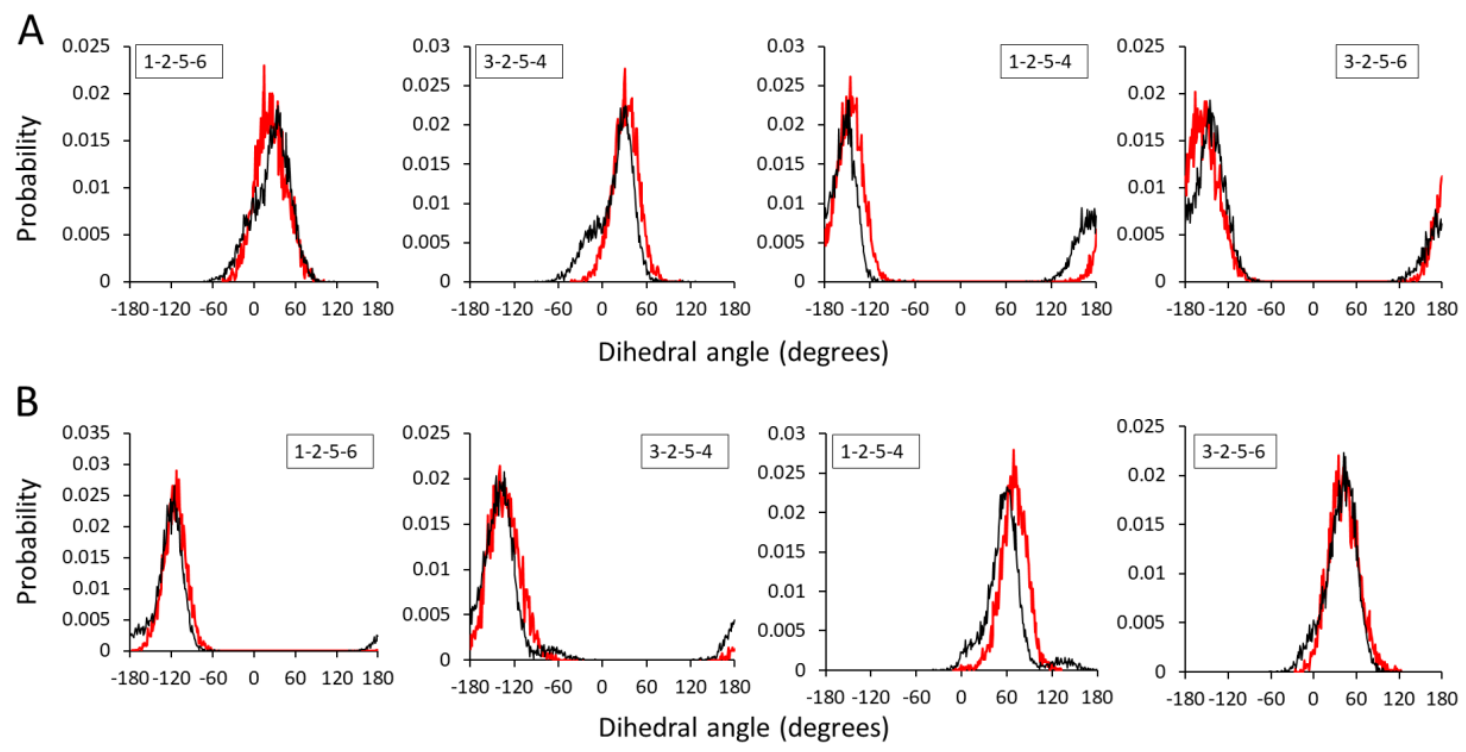

Figure 4. Dihedral angle distributions of AA and CG representations for (A) HA1-3 and (B) HA1-4. Black and red lines indicate AA and CG representations, respectively.

\subsubsection{Global Properties}

Two important global properties of the HA polymer, namely the end-to-end distance $\left(R_{e}\right)$ and radius of gyration $\left(\mathrm{R}_{\mathrm{g}}\right)$, were calculated as a part of the validation and application of our CG model for HA (Figure 5). The parameters obtained from the CG modeling of HA1-3 and HA1-4 dimers were applied to model a larger HA polymer having eight monosaccharide subunits [42]. The results were compared to test if the CG model can reproduce the global properties by taking AA data as a reference. Additional MC angles and dihedral angles were applied in the case of HA octasaccharide. The distributions are indicated in Figures 6 and 7 . The $R_{e}$ value of the HA polymer was calculated as the average distance between the MC beads 2 and 23 and matched with the $R_{e}$ obtained from the analogous averaged AA simulations (Figure $2 B$ ). The $R_{e}$ of HA polymers were well matched where the average lengths observed were $33.19 \AA$ and $30.52 \AA$ for AA and CG models, respectively (Figure 5A). The $\mathrm{R}_{\mathrm{g}}$ of HA polymers overlapped well, with average values of $1.12 \mathrm{~nm}$ and $1.07 \mathrm{~nm}$ for AA and CG models, respectively (Figure 5B). An HA molecule eight monomers in length was used to obtain the $\mathrm{R}_{\mathrm{g}}$ value, which is too short when directly compared with experimental $R_{g}$. Therefore, we performed another simulation for HA comprised of 100 monomers in $150 \mathrm{mM}$ of $\mathrm{NaCl}$ to correlate $\mathrm{R}_{\mathrm{g}}$ values with experimental results reported by Mendichi et al. [45]. The experimental results of $R_{\mathrm{g}}$ followed power law as a function of molar weight in the above reference report. This experimental results estimate $\mathrm{R}_{\mathrm{g}}$ of HA as $9.4 \mathrm{~nm}$ while we obtained a value of $8.1 \mathrm{~nm}$. We believe that our simulation results are comparable to the experiment. Furthermore, the CG model of HA showed a very low range of the RMSD values of $0.005 \mathrm{~nm}$ to $0.498 \mathrm{~nm}$, indicating an overall stiff structure of the HA (Figure 5C) [46]. The time-averaged RMSD values show a very close match, with values of 0.28 for AA and 0.31 and CG. These results confirm that the structure of CG-modeled HA is stiff in nature, which is comparable to 
AA HA polymer. Evidently, CG simulations of HA models have produced results that match well with the AA simulations.
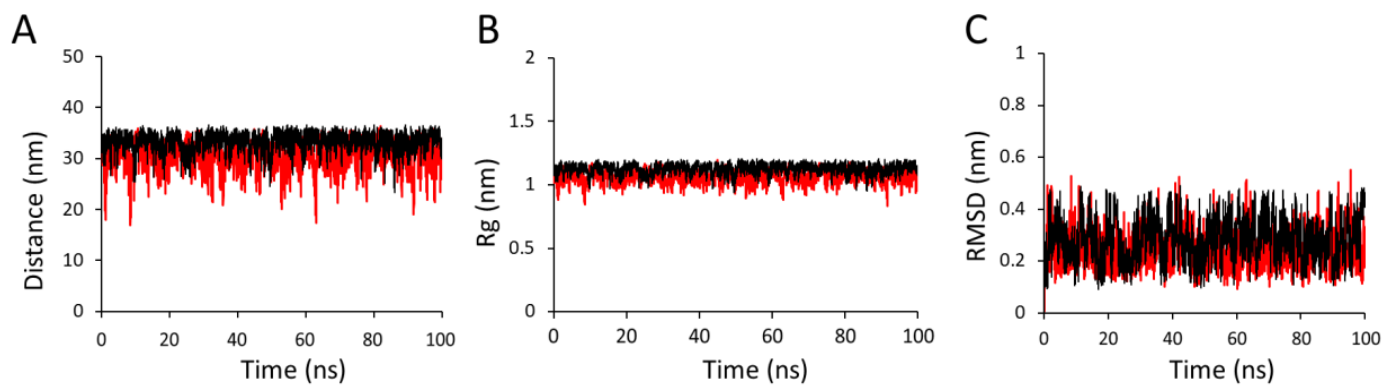

Figure 5. Global properties of an HA octasaccharide molecule: (A) End-to-end distance $\left(\mathrm{R}_{\mathrm{e}}\right)$, (B) Radius of gyration $\left(\mathrm{R}_{\mathrm{g}}\right)$, and $(\mathrm{C})$ root mean square deviation (RMSD). Black and red lines indicate AA and CG representations, respectively.
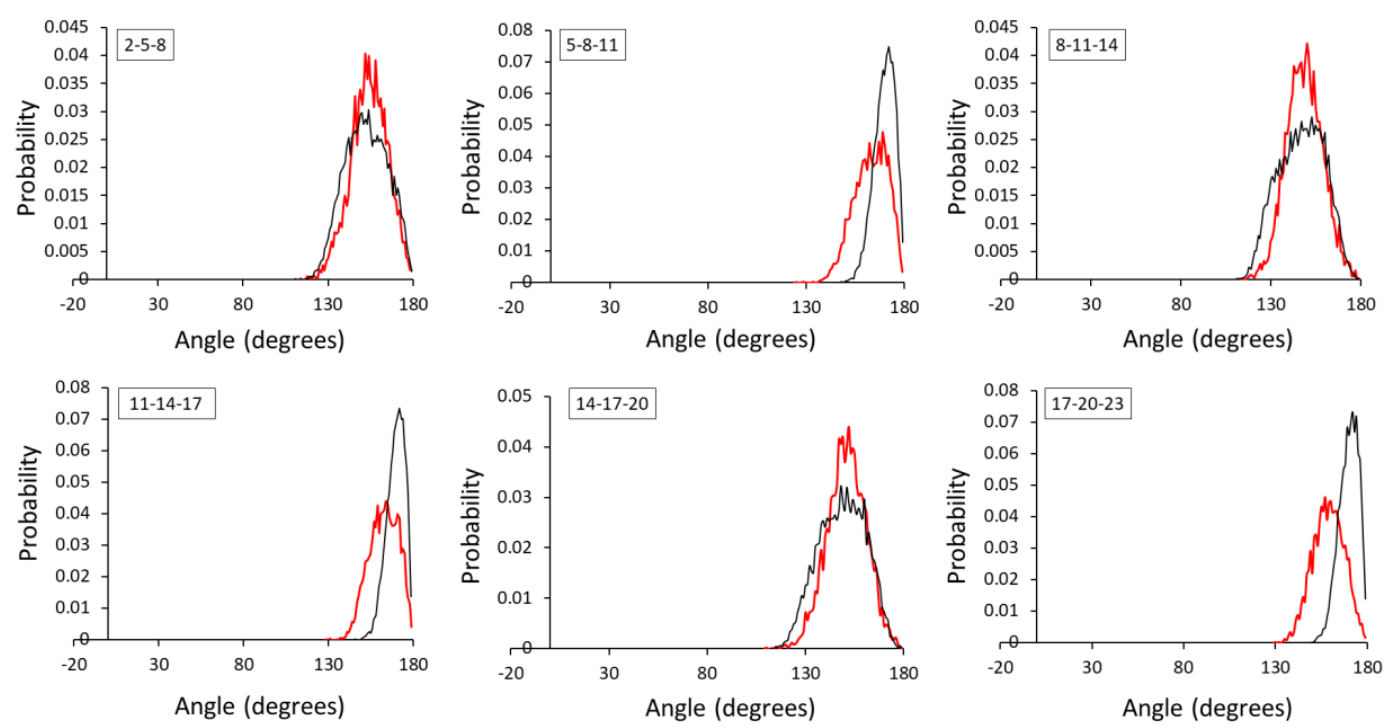

Figure 6. Main chain angle distributions of AA and CG representations for an HA octasaccharide molecule. Black and red lines indicate AA and CG representations, respectively.
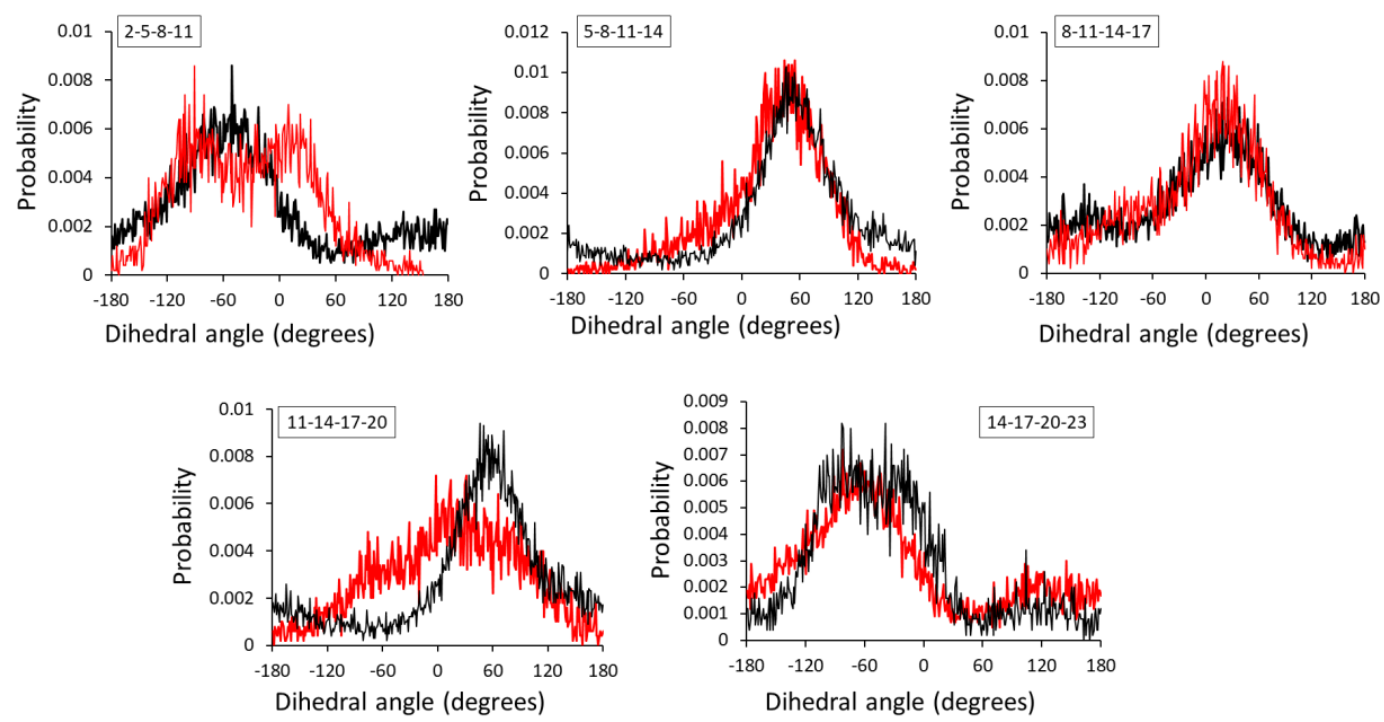

Figure 7. Main chain dihedral angle distributions of AA and CG representations for an HA octasaccharide molecule. Black and red lines indicate AA and CG representations, respectively. 


\subsection{Condensation of the Gel}

\subsubsection{HA Gel Formation}

We applied the coarse-grained model to the simulation of mesoscopic gel formation. Because HA is a negatively charged polymer, it will not aggregate unless the strong electrostatic repulsion is regulated. This is not feasible in AA because the mesoscopic phenomenon requires the consideration of multiple chains in mesoscale, and water molecules should be considered explicitly since the part of the attraction comes from the hydrophobic interaction and hydrogen bond interaction. In the simulation, we considered 16 HA chains and each was comprised of 100 monomers or 50 dimeric repeating units. The volume fraction of HA was set to be 1/1000 which corresponds to the swelling states for weakly charged polyelectrolytes [9]. In addition to the HAs and water, we added $100 \mathrm{mM}$ $\mathrm{NaCl}$ as a physiological condition. As seen in Figure 8, we can observe that HAs are inter-connected to form a gel phase overcoming the long-range electrostatic repulsion. The crosslink is due to the short-ranged attraction from the combination of van der Waals, hydrophobic interactions, and hydrogen bonds. The result is consistent with the experimental finding of HA gel formation in physiological conditions [10], which verifies the validity of our model and its usefulness.
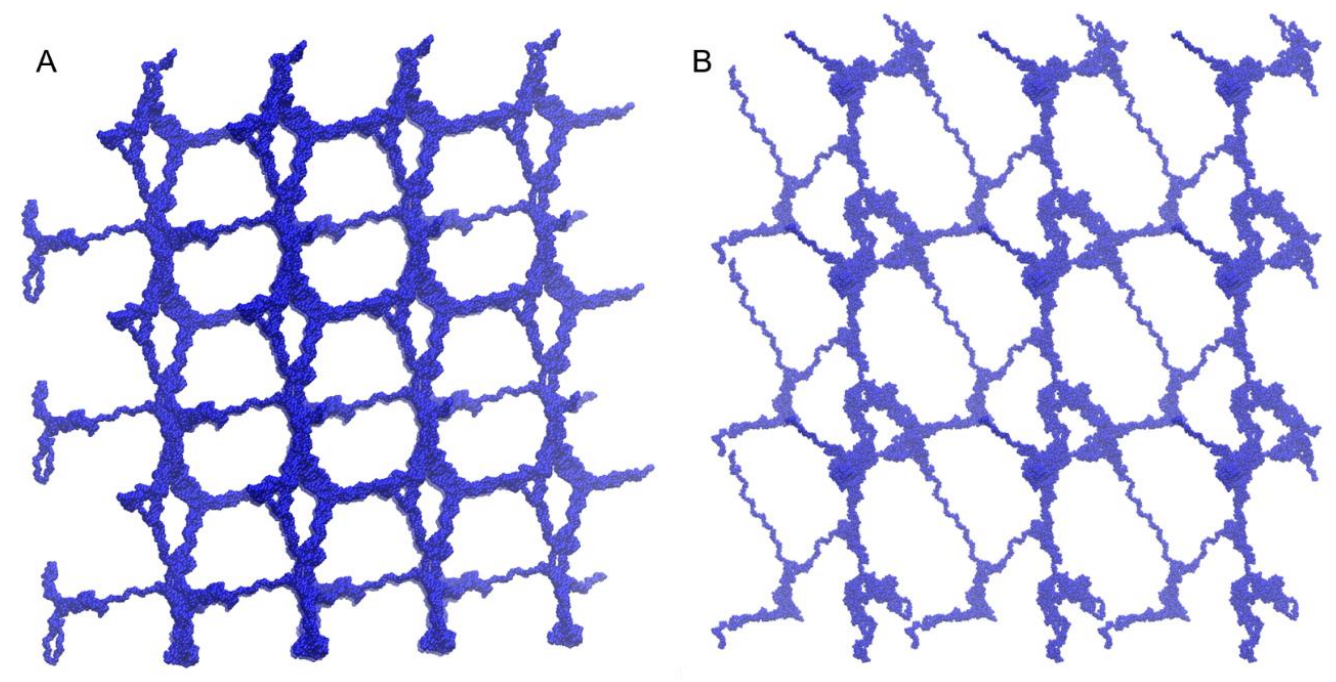

Figure 8. The simulation configuration of $\mathrm{HA}$ gel in $100 \mathrm{mM} \mathrm{NaCl}$ and: (A) $0 \mathrm{mM} \mathrm{CaCl}_{2}$; (B) $200 \mathrm{mM} \mathrm{CaCl}_{2}$.

\subsubsection{Swelling of the HA Gel in the Presence of Monovalent Salt and Divalent Salt}

With the concentration of $\mathrm{NaCl}$ fixed at $100 \mathrm{mM}$, we changed the concentration of $\mathrm{CaCl}_{2}$ from 0 to $100 \mathrm{mM}$. Throughout the whole range of the concentration of $\mathrm{CaCl}_{2}$, the gel was still swollen, unlike other biopolyelectrolytes such as DNA. This is also consistent with the experimental results [10]. It is clear that $\mathrm{NaCl}$ screens the electrostatic interaction between HA chains. In order to investigate the change of the microscopic structure, we measured the surface area of the polyelectrolyte network. The surface area will be decreased significantly if polymers are collapsed. As seen in Figure 9A, the area increased at high $\mathrm{CaCl}_{2}$ concentration, but the change was not drastic. There seemed to be a slight change in the local aggregation at cross-links, but a substantial change in the network structure was not identified.

In the experiment, the osmotic pressure was reduced with the addition of divalent salts. The polymeric contribution to the osmotic pressure of the gel corresponds to the energy stored in the volume of the network mesh (Equation (3)). That is,

$$
P_{o s m}=\frac{k_{B} T}{l^{3}},
$$


where $l$ is the length of the network mesh. The length of the network mesh of the polyelectrolyte gel is proportional to $l \propto I^{1 / 4}$, where $I=\frac{1}{2} \sum z_{i} c_{s i}^{2}$ at the condition that $c_{s+}, c_{s_{-}} \gg c$ for salt concentration $c_{s+}, c_{S-}$ of positive and negative salt ions, and the polyelectrolyte concentration $c$. Then, the osmotic pressure is given by the following equation (Equation (4))

$$
P_{\text {osm }} \propto I^{-\frac{3}{4}}
$$

Thus, the addition of $\mathrm{CaCl}_{2}$ increases $I$ which results in the decrease of the osmotic pressure in the experiments [10]. In our simulation, we did not measure the osmotic pressure directly, but we saw the consistent trend that the number of crosslinks seemed to decrease at a high salt concentration of $\mathrm{CaCl}_{2}$ (Figure 9).

Next we reduced the concentration of $\mathrm{NaCl}$ to $40 \mathrm{mM}$ (Figure 9B). At this salt concentration some polyelectrolytes such as sodium polyacrylate undergo a swollen to collapsed transition under high $\mathrm{CaCl}_{2}$ concentration due to the ion-bridging of divalent $\mathrm{Ca}^{2+}[9]$. However, our results consistently show that $\mathrm{HA}$ still remained in the swollen phase even for this low $\mathrm{NaCl}$ concentration, without establishing any ionic bridging or the resultant collapse of the gel. This stability of the HA gel under various salt concentrations is a merit for biological and medical applications.
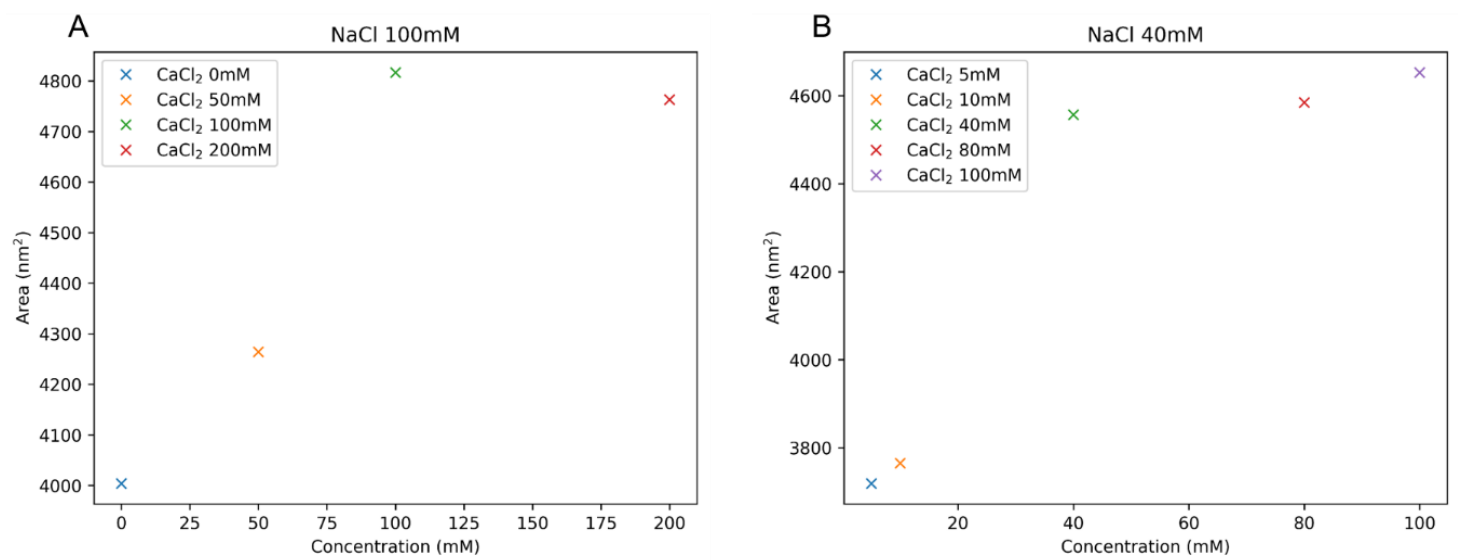

Figure 9. Area of $\mathrm{HA}$ gel when the concentrations of $\mathrm{NaCl}$ are (A) $100 \mathrm{mM}$ and (B) $40 \mathrm{mM}$. The area is plotted as a function of $\mathrm{CaCl}_{2}$ concentration.

\section{Conclusions}

We developed a CG model of HA using the Martini scheme and compared its performance with all-atom simulations. We chose to model the HA1-3 and HA1-4 bonding disaccharide units separately. The molecular masses of the CG beads were adapted according to their AA analogs to achieve realistic simulations results. Our HA CG model was able to accurately reproduce the probability distributions for bonded interactions when compared to all-atom simulations. The obtained parameters were applied to an octasaccharide HA polymer in order to assess the performance of our CG model. The results of polymer global properties such as $R_{e}$ and $R_{g}$ were in good agreement with atomistic data. The structure of the HA polymer is unusually stiff in aqueous solution, which is indicated by low RMSD values during atomistic simulations. Similar results were obtained for our CG model of HA, which indicates its usefulness for modeling long HA polymers. Consequently, the CG modeling technique presented here makes long HA polymers with high molecular weights within reach in the molecular dynamics simulations approach. Here we applied our model to HA gel formation, which is usually intractable in atomistic molecular dynamics. We investigated the HA gel formation in various mixtures of $\mathrm{NaCl}$ monovalent salt and $\mathrm{CaCl}_{2}$ divalent salt, from physiological to lab conditions. We found that HA formed a stable swollen gel over the salt concentrations we investigated, which is consistent with the experimental results. This is a distinguishable feature of HA from regular 
proteins, whose backbones are usually hydrophobic. This property is advantageous for bioengineering applications of HA. We believe these developed procedures could serve as a useful reference for further coarse-grained modeling of other polyelectrolytes in order to enable mesoscale simulations with large molecules. Our model developed here can be used in conjunction with other Martini models, and can be further expanded to model various HA polymers and their modified polyelectrolytes, for example, the process of physical crosslinking of HA in the presence of phospholipids (HA-PL complex) in the lubrication of articular cartilage [47,48], self-assembly of polymeric nanoparticles in drug delivery $[49,50]$, novel HA-based biocompatible and biodegradable hydrogels for in vitro cell culture [2,51], cartilage tissue engineering [3], and the charge-based purification of protein drugs [4].

\section{Materials and Methods}

\subsection{Model}

A typical Martini model applies a mapping of four heavy atoms and associated hydrogens to one (4:1 mapping) CG bead [52]. Each CG bead is assigned a bead type based on thermodynamic properties obtained from experiments and simulations of its AA molecular analog. Different bead sizes can be used including a "standard" bead representing 4:1 mapping. "Small" and "tiny" beads with 3:1 or 2:1 mapping are typically used to model ring-like structures and base stacking in nucleic acids. The mapping strategy is flexible and can be adjusted depending on one's need such that higher or lower or even fractional mapping ratios can be used for different biomolecules [53]. Furthermore, Martini models are kept simple by assigning only four main types of interaction sites or bead types such as polar $(\mathrm{P})$, nonpolar $(\mathrm{N})$, apolar $(\mathrm{C})$, and charged $(\mathrm{Q})$ beads. The Martini "standard" CG beads types can be further subdivided to capture their different chemical natures. Depending on their polarity, polar and apolar bead types are subdivided into five levels from 1 to 5 (polarity low to high). Whereas, nonpolar and charged beads are subdivided based on their hydrogen bonding ( $\mathrm{d}$ (donor), a (acceptor), da (both), 0 (none)) capability. The parameters for nonbonded interactions are determined by the assigned bead type and size. The mass of CG beads can be adapted according to its AA analog in order to achieve realistic simulations [52]. For bonded parameters, two sets of simulations (AA and CG) are run and probability distributions of bond lengths, bond angles, and dihedral angles are compared and tuned by trial and error until a good match is obtained.

\subsection{CG Mapping}

The HA molecule was mapped based on the Martini model for carbohydrates where disaccharides are modeled as two three-bead units connected by a single bond mimicking the glycosidic linkage in carbohydrates [43]. Such additional bonds are used to connect multiple monosaccharides units to construct oligosaccharide and polysaccharide CG mappings. We parameterized HA1-3 and HA1-4 subunits separately (Figure 1A,B). Two types of Martini CG bead types were assigned: polar (P type) and charged (Q type) (Tables 1 and 2). In the case of GlcA, the charged bead type was Qa, while the remaining two were assigned P2 and $\mathrm{P} 4$ bead types. In GlcNAc, all three beads were polar and included two P2 and one P1 bead type. This type of mapping was carried forward to a larger molecule of HA with eight monosaccharide subunits whose structure (PDB ID: 2BVK) is available at the RCSB database [42]. We denoted the central beads of both monosaccharide units 2, 5, 8, 11, 14, 17, 20, and 23 as main chain (MC), atoms which are represented by the P2 bead type (Figure 1C). 
Table 1. Assignment of Martini CG bead types for the HA1-3 disaccharide.

\begin{tabular}{|c|c|c|c|c|}
\hline Bonding & Structure & No. & Bead Name & Bead Type \\
\hline \multirow{6}{*}{ GlcA1-3GlcNAc } & & 1 & B1 & $\mathrm{Qa}$ \\
\hline & & 2 & B2 & $\mathrm{P} 2$ \\
\hline & & 3 & B3 & P4 \\
\hline & & 4 & B4 & P2 \\
\hline & & 5 & B5 & P2 \\
\hline & & 6 & B6 & P1 \\
\hline
\end{tabular}

Table 2. Assignment of Martini CG bead types for HA1-4 disaccharide.

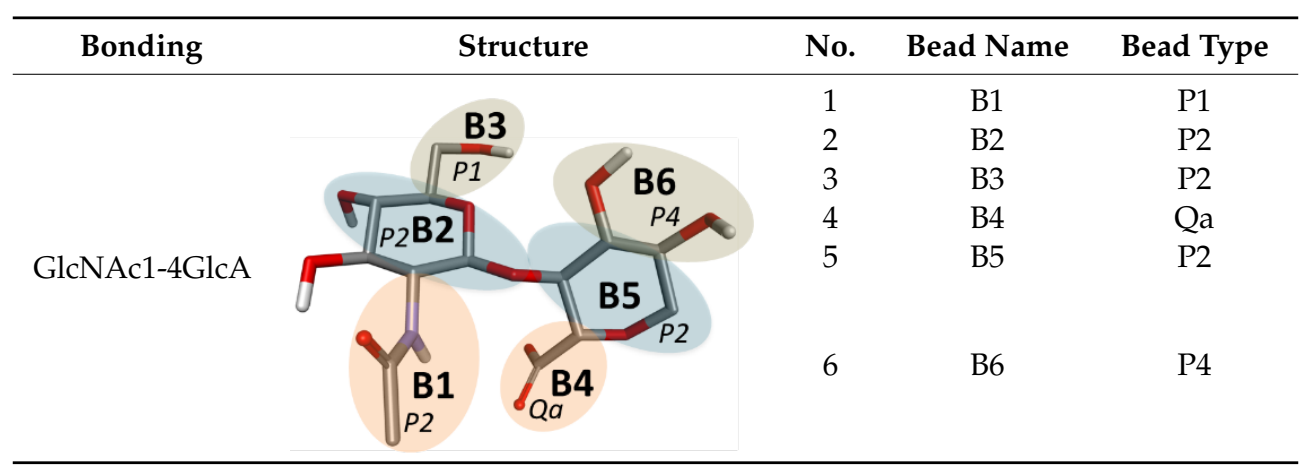

\subsection{Non-Bonded Interactions}

The non-bonded interactions were mainly represented by van der Waals interactions and electrostatic interactions (Equation (5)). The van der Waals interactions were described by the 12-6 Lennard-Jones (LJ) potential energy function given as

$$
V_{L J}=4 \varepsilon_{i j}\left[\left(\frac{\sigma_{i j}}{r_{i j}}\right)^{12}-\left(\frac{\sigma_{i j}}{r_{i j}}\right)^{6}\right]
$$

where $\sigma_{i j}$ represents the closest distance of approach between two particles while the strength of their interaction is given by $\varepsilon_{i j}$, and the LJ parameters $\sigma_{i j}$ and $\varepsilon_{i j}$ were determined by the assigned bead type. In case of the charged beads, the electrostatic interaction was represented by the Columbic potential energy function (Equation (6)) given as

$$
V_{e l}=\frac{q_{i} q_{j}}{4 \pi \varepsilon_{0} \varepsilon_{r} r_{i j}}
$$

where $q_{i}$ and $q_{j}$ denote the charges of CG beads $i$ and $j$, respectively, $\varepsilon_{r}$ is the relative dielectric constant of the solvent, and $\varepsilon_{0}$ is the permittivity of free space.

\subsection{Bonded Interactions}

The bonded interactions mainly constituted bond lengths, bond angles, and dihedral angles. The harmonic potential $V_{b}$ is used to model bond lengths (Equation (7)). Bond length parameter $r_{i j}$ denotes the distance between two bonded beads $i$ and $j, r_{e q}$ is the equilibrium bond length, and $\mathrm{K}_{\mathrm{b}}$ represents the force constant.

$$
V_{b}=\frac{1}{2} K_{b}\left(r_{i j}-r_{e q}\right)^{2}
$$


A cosine type harmonic potential denoted by $V_{a}$ was used to model bond angles (Equation (8)), where $\theta_{i j k}$ is the angle between three consecutive beads $i, j$, and $k, \theta_{e q}$ is the equilibrium bond angle, and $\mathrm{K}_{\mathrm{a}}$ is the force constant.

$$
V_{a}=\frac{1}{2} K_{a}\left(\cos \left(\theta_{i j k}\right)-\cos \left(\theta_{e q}\right)\right)^{2}
$$

$V_{d}$ represents a sum of periodic potentials where $\varphi_{i j k l}$ is the dihedral angle between sequential beads $i, j, k$, and $l$ (Equation (9)). The force constant for dihedral angles is given by $K_{\mathrm{d}}$, number of peaks in this potential by $n$, and $\varphi_{d}$ specifies the location of peaks in periodic potential.

$$
V_{d}=K_{d}\left(1+\cos \left(n \varphi_{i j k l}-\varphi_{d}\right)\right)
$$

\subsection{AA Simulations}

AA simulations were run on three different molecules: two HA dimers HA1-3 and HA1-4, and an HA octasaccharide, independently. The simulations were carried out with CHARMM36 force field using Groningen machine for chemical simulations (GROMACS 2018) software [54]. The topology files for all ligands were generated using the CHARMM General Force Field (CGenFF) program implemented in CHARMM-GUI webserver [55]. Each system contained one HA in dodecahedron water box of thickness $10 \AA$ containing TIP3P waters with an appropriate number of counter-ions added to neutralize the system. The initial structures were relaxed through 50,000 steps of energy minimization with a maximum force of $1000 \mathrm{~kJ} / \mathrm{mol}$ using steepest descent algorithm. Thereafter, a two-step equilibration procedure including a constrained NVT simulation at $300 \mathrm{~K}$ and a constrained NPT simulation at $300 \mathrm{~K}$ and 1 bar was performed. The temperature was maintained using a V-rescale thermostat with a time constant of 0.1 ps. The Parrinello-Rahman barostat was used to maintain the pressure with a time constant of $2 \mathrm{ps}$ and compressibility of $4.5 \times 10^{-5} \mathrm{bar}^{-1}$. The bonds were constrained using the LINCS algorithm. An unrestrained production run was carried out for $100 \mathrm{~ns}$ under NPT conditions using periodic boundary condition applied in all directions. The equations of motion were integrated using the leapfrog algorithm with a time step of $2 \mathrm{fs}$. The long-range electrostatic interactions were calculated using the particle mesh Ewald (PME) method. Nonbonded short-range interactions were cut off at $1.0 \mathrm{~nm}$.

\subsection{CG Simulations}

The average structures were taken from the last $50 \mathrm{~ns}$ of AA simulations. These equilibrated configurations were used to generate initial structures of HAs for CG simulations using the mapping scheme specified in the CG mapping section described above. The same temperature and pressure conditions were maintained similar as in the AA simulations. Solvation was done with a standard non-polarizable water model and counterions were added to neutralize the system. Further, the energy minimization was followed by a constrained NPT equilibration of $1 \mathrm{~ns}$, excluding NVT. The NVT simulation was not necessary prior to the NPT simulation as the low-amplitude high-frequency fluctuations were removed from the potential energy surfaces (PES) in the CG models [14]. The cut-off value of $1.1 \mathrm{~nm}$ was used for the LJ potential and short-range nonbonded interactions. The dielectric constant of 15 was used for water. A comparatively larger time step of $10 \mathrm{fs}$ was used in all CG simulations compared to AA simulations. A production run of $100 \mathrm{~ns}$ was completed under the unconstrained NPT simulation with periodic boundary conditions applied in all directions.

Author Contributions: Conceptualization, R.K. and Y.J.; simulation, R.K. and Y.L.; analysis, R.K., Y.L., and Y.J.; writing, R.K. and Y.J.; project administration, Y.J.; funding acquisition, Y.J. All authors have read and agreed to the published version of the manuscript.

Funding: This work was supported by Creative Materials Discovery Program through the National Research Foundation of Korea (NRF) funded by the Ministry of Science and ICT (NRF-2018M3D1A1058624) and NRF-2018R1A2B6006262.

Conflicts of Interest: The authors declare no conflict of interest. 


\section{References}

1. Laurent, T.C.; Fraser, J.R. Hyaluronan. FASEB J. 1992, 6, 2397-2404. [CrossRef] [PubMed]

2. Burdick, J.A.; Prestwich, G.D. Hyaluronic Acid Hydrogels for Biomedical Applications. Adv. Mater. 2011, 23, H41-H56. [CrossRef] [PubMed]

3. Collins, M.N.; Birkinshaw, C. Hyaluronic Acid Based Scaffolds for Tissue Engineering-A Review. Carbohydr. Polym. 2013, 92, 1262-1279. [CrossRef] [PubMed]

4. Du, X.; Dubin, P.L.; Hoagland, D.A.; Sun, L. Protein-Selective Coacervation with Hyaluronic Acid. Biomacromolecules 2014, 15, 726-734. [CrossRef] [PubMed]

5. Hill, T.L. Some Statistical Mechanical Models of Elastic Polyelectrolytes and Proteins. J. Chem. Phys. 1952, 20, 1259-1273. [CrossRef]

6. Tasaki, I.; Byrne, P.M. Discontinuous volume transitions in ionic gels and their possible involvement in the nerve excitation process. Biopolymers 1992, 32, 1019-1023. [CrossRef] [PubMed]

7. Zhang, Y.; Douglas, J.F.; Ermi, B.D.; Amis, E.J. Influence of counterion valency on the scattering properties of highly charged polyelectrolyte solutions. J. Chem. Phys. 2001, 114, 3299-3313. [CrossRef]

8. Horkay, F.; Tasaki, I.; Basser, P.J. Osmotic swelling of polyacrylate hydrogels in physiological salt solutions. Biomacromolecules 2000, 1, 84-90. [CrossRef]

9. Yin, D.W.; Horkay, F.; Douglas, J.F.; de Pablo, J.J. Molecular simulation of the swelling of polyelectrolyte gels by monovalent and divalent counterions. J. Chem. Phys. 2008, 129, 154902. [CrossRef]

10. Horkay, F.; Basser, P.J.; Londono, D.J.; Hecht, A.M.; Geissler, E. Ions in hyaluronic acid solutions. J. Chem. Phys. 2009, 131, 184902. [CrossRef]

11. Timilsena, Y.P.; Akanbi, T.O.; Khalid, N.; Adhikari, B.; Barrow, C.J. Complex Coacervation: Principles, Mechanisms and Applications in Microencapsulation. Int. J. Biol. Macromol. 2019, 121, 1276-1286. [CrossRef]

12. Lee, J.; Popov, Y.O.; Fredrickson, G.H. Complex Coacervation: A Field Theoretic Simulation Study of Polyelectrolyte Complexation. J. Chem. Phys. 2008, 128, 224908. [CrossRef]

13. Danielsen, S.P.O.; McCarty, J.; Shea, J.E.; Delaney, K.T.; Fredrickson, G.H. Molecular Design of Self-Coacervation Phenomena in Block Polyampholytes. Proc. Natl. Acad. Sci. USA 2019, 116, 8224-8232. [CrossRef]

14. Noid, W.G. Perspective: Coarse-grained models for biomolecular systems. J. Chem. Phys. 2013, $139,090901$. [CrossRef]

15. Fritz, D.; Harmandaris, V.A.; Kremer, K.; van der Vegt, N.F.A. Coarse-Grained Polymer Melts Based on Isolated Atomistic Chains: Simulation of Polystyrene of Different Tacticities. Macromolecules 2009, 42, 7579-7588. [CrossRef]

16. Rossi, G.; Monticelli, L.; Puisto, S.R.; Vattulainen, I.; Ala-Nissila, T. Coarse-graining polymers with the MARTINI force-field: Polystyrene as a benchmark case. Soft Matter. 2011, 7, 698-708. [CrossRef]

17. Panizon, E.; Bochicchio, D.; Monticelli, L.; Rossi, G. MARTINI Coarse-Grained Models of Polyethylene and Polypropylene. J. Phys. Chem. B 2015, 119, 8209-8216. [CrossRef]

18. Bochicchio, D.; Panizon, E.; Monticelli, L.; Rossi, G. Interaction of hydrophobic polymers with model lipid bilayers. Sci. Rep. 2017, 7, 6357. [CrossRef] [PubMed]

19. Nawaz, S.; Carbone, P. Coarse-graining poly(ethylene oxide)-poly(propylene oxide)-poly(ethylene oxide) (PEO-PPO-PEO) block copolymers using the MARTINI force field. J. Phys. Chem. B 2014, 118, 1648-1659. [CrossRef] [PubMed]

20. Pérez-Sánchez, G.; Vicente, F.A.; Schaeffer, N.; Cardoso, I.S.; Ventura, S.P.M.; Jorge, M.; Coutinho, J.A.P. Rationalizing the Phase Behavior of Triblock Copolymers through Experiments and Molecular Simulations. J. Phys. Chem. C 2019, 123, 21224-21236. [CrossRef]

21. Ramezanghorbani, F.; Lin, P.; Colina, C.M. Optimizing Protein-Polymer Interactions in a Poly(ethylene glycol) Coarse-Grained Model. J. Phys. Chem. B 2018, 122, 7997-8005. [CrossRef] [PubMed]

22. Salassi, S.; Simonelli, F.; Bartocci, A.; Rossi, G. A Martini coarse-grained model of the calcein fluorescent dye. J. Phys. D Appl. Phys. 2018, 51, 384002. [CrossRef]

23. Mahajan, S.; Tang, T. Martini Coarse-Grained Model for Polyethylenimine. J. Comput. Chem. 2019, 40, 607-618. [CrossRef]

24. Khan, P.; Goel, G. Martini Coarse-Grained Model for Clay-Polymer Nanocomposites. J. Phys. Chem. B 2019, 123, 9011-9023. [CrossRef] 
25. Rolland, N.; Modarresi, M.; Franco-Gonzalez, J.F.; Zozoulenko, I. Large scale mobility calculations in PEDOT (Poly(3,4-ethylenedioxythiophene)): Backmapping the coarse-grained MARTINI morphology. Comput. Mater. Sci. 2020, 179, 109678. [CrossRef]

26. Bochicchio, D.; Pavan, G.M. Effect of Concentration on the Supramolecular Polymerization Mechanism via Implicit-Solvent Coarse-Grained Simulations of Water-Soluble 1,3,5-Benzenetricarboxamide. J. Phys. Chem. Lett. 2017, 8, 3813-3819. [CrossRef] [PubMed]

27. Bochicchio, D.; Pavan, G.M. Molecular modelling of supramolecular polymers. Adv. Phys. X 2018, 3, 1436408 . [CrossRef]

28. Lee, O.S.; Cho, V.; Schatz, G.C. Modeling the self-assembly of peptide amphiphiles into fibers using coarse-grained molecular dynamics. Nano Lett. 2012, 12, 4907-4913. [CrossRef]

29. Manandhar, A.; Kang, M.; Chakraborty, K.; Tang, P.K.; Loverde, S.M. Molecular simulations of peptide amphiphiles. Org. Biomol. Chem. 2017, 15, 7993-8005. [CrossRef]

30. Bruininks, B.M.H.; Souza, P.C.T.; Marrink, S.J. A Practical View of the Martini Force Field. Methods Mol. Biol. 2019, 2022, 105-127.

31. Grunewald, F.; Rossi, G.; de Vries, A.H.; Marrink, S.J.; Monticelli, L. Transferable MARTINI Model of Poly(ethylene Oxide). J. Phys. Chem. B 2018, 122, 7436-7449. [CrossRef] [PubMed]

32. Huang, H.; Wu, L.; Xiong, H.; Sun, H. A Transferrable Coarse-Grained Force Field for Simulations of Polyethers and Polyether Blends. Macromolecules 2019, 52, 249-261. [CrossRef]

33. Kuo, A.-T.; Okazaki, S.; Shinoda, W. Transferable coarse-grained model for perfluorosulfonic acid polymer membranes. J. Chem. Phys. 2017, 147, 094904. [CrossRef] [PubMed]

34. Campos-Villalobos, G.; Siperstein, F.R.; Patti, A. Transferable coarse-grained MARTINI model for methacrylate-based copolymers. Mol. Syst. Des. Eng. 2019, 4, 186-198. [CrossRef]

35. Rodgers, J.M.; Sorensen, J.; de Meyer, F.J.; Schiott, B.; Smit, B. Understanding the phase behavior of coarse-grained model lipid bilayers through computational calorimetry. J. Phys. Chem. B 2012, 116, 1551-1569. [CrossRef]

36. Waheed, Q.; Tjornhammar, R.; Edholm, O. Phase transitions in coarse-grained lipid bilayers containing cholesterol by molecular dynamics simulations. Biophys. J. 2012, 103, 2125-2133. [CrossRef]

37. Wang, Y.; Gkeka, P.; Fuchs, J.E.; Liedl, K.R.; Cournia, Z. DPPC-cholesterol phase diagram using coarse-grained Molecular Dynamics simulations. Biochim. Et Biophys. Acta 2016, 1858, 2846-2857. [CrossRef]

38. Winands, T.; Bockmann, M.; Schemme, T.; Ly, P.M.; de Jong, D.H.; Wang, Z.; Denz, C.; Heuer, A.; Doltsinis, N.L. P3HT:DiPBI bulk heterojunction solar cells: Morphology and electronic structure probed by multiscale simulation and UV/vis spectroscopy. Phys. Chem. Chem. Phys PCCP 2016, 18, 6217-6227. [CrossRef]

39. Larsson, P.; Alskar, L.C.; Bergstrom, C.A.S. Molecular Structuring and Phase Transition of Lipid-Based Formulations upon Water Dispersion: A Coarse-Grained Molecular Dynamics Simulation Approach. Mol. Pharm. 2017, 14, 4145-4153. [CrossRef]

40. Wei, Z.; Li, S.; Ning, N.; Tian, M.; Zhang, L.; Mi, J. Theoretical and Experimental Insights into the Phase Transition of Rubber/Plastic Blends during Dynamic Vulcanization. Ind. Eng. Chem. Res. 2017, 56, 13911-13918. [CrossRef]

41. Carpenter, T.S.; Lopez, C.A.; Neale, C.; Montour, C.; Ingolfsson, H.I.; Di Natale, F.; Lightstone, F.C.; Gnanakaran, S. Capturing Phase Behavior of Ternary Lipid Mixtures with a Refined Martini Coarse-Grained Force Field. J. Chem. Theory Comput. 2018, 14, 6050-6062. [CrossRef]

42. Almond, A.; Deangelis, P.L.; Blundell, C.D. Hyaluronan: The Local Solution Conformation Determined by Nmr and Computer Modeling Is Close to a Contracted Left-Handed 4-Fold Helix. J. Mol. Biol. 2006, 358, 1256-1269. [CrossRef]

43. Lopez, C.A.; Rzepiela, A.J.; de Vries, A.H.; Dijkhuizen, L.; Hunenberger, P.H.; Marrink, S.J. Martini Coarse-Grained Force Field: Extension to Carbohydrates. J. Chem. Theory Comput. 2009, 5, 3195-3210. [CrossRef]

44. Uusitalo, J.J.; Ingolfsson, H.I.; Akhshi, P.; Tieleman, D.P.; Marrink, S.J. Martini Coarse-Grained Force Field: Extension to DNA. J. Chem. Theory Comput. 2015, 11, 3932-3945. [CrossRef] [PubMed]

45. Mendichi, R.; Soltes, L.; Giacometti Schieroni, A. Evaluation of radius of gyration and intrinsic viscosity molar mass dependence and stiffness of hyaluronan. Biomacromolecules 2003, 4, 1805-1810. [CrossRef] [PubMed]

46. Almond, A.; Sheehan, J.K.; Brass, A. Molecular Dynamics Simulations of the Two Disaccharides of Hyaluronan in Aqueous Solution. Glycobiology 1997, 7, 597-604. [CrossRef] 
47. Dedinaite, A.; Claesson, P.M. Synergies in Lubrication. Phys. Chem. Chem. Phys. 2017, 19, 23677-23689. [CrossRef]

48. Siodmiak, J.; Beldowski, P.; Auge, W.K.; Ledzinski, D.; Smigiel, S.; Gadomski, A. Molecular Dynamic Analysis of Hyaluronic Acid and Phospholipid Interaction in Tribological Surgical Adjuvant Design for Osteoarthritis. Molecules 2017, 22, 1436. [CrossRef]

49. Svechkarev, D.; Kyrychenko, A.; Payne, W.M.; Mohs, A.M. Probing the Self-Assembly Dynamics and Internal Structure of Amphiphilic Hyaluronic Acid Conjugates by Fluorescence Spectroscopy and Molecular Dynamics Simulations. Soft Matter. 2018, 14, 4762-4771. [CrossRef]

50. Min, Q.; Yu, X.; Liu, J.; Wu, J.; Wan, Y. Chitosan-Based Hydrogels Embedded with Hyaluronic Acid Complex Nanoparticles for Controlled Delivery of Bone Morphogenetic Protein-2. Pharmaceutics 2019, 11, 214. [CrossRef]

51. Shu, X.Z.; Liu, Y.; Palumbo, F.; Prestwich, G.D. Disulfide-Crosslinked Hyaluronan-Gelatin Hydrogel Films: A Covalent Mimic of the Extracellular Matrix for in Vitro Cell Growth. Biomaterials 2003, 24, 3825-3834. [CrossRef]

52. Marrink, S.J.; Risselada, H.J.; Yefimov, S.; Tieleman, D.P.; de Vries, A.H. The Martini Force Field: Coarse Grained Model for Biomolecular Simulations. J. Phys. Chem. B 2007, 111, 7812-7824. [CrossRef] [PubMed]

53. Marrink, S.J.; de Vries, A.H.; Mark, A.E. Coarse Grained Model for Semiquantitative Lipid Simulations. J. Phys. Chem. B 2004, 108, 750-760. [CrossRef]

54. Abraham, M.J.; Murtola, T.; Schulz, R.; Páll, S.; Smith, J.C.; Hess, B.; Lindahl, E. GROMACS: High performance molecular simulations through multi-level parallelism from laptops to supercomputers. SoftwareX 2015, 1-2, 19-25. [CrossRef]

55. Jo, S.; Kim, T.; Iyer, V.G.; Im, W. Charmm-Gui: A Web-Based Graphical User Interface for Charmm. J. Comput. Chem. 2008, 29, 1859-1865. [CrossRef]

(C) 2020 by the authors. Licensee MDPI, Basel, Switzerland. This article is an open access article distributed under the terms and conditions of the Creative Commons Attribution (CC BY) license (http://creativecommons.org/licenses/by/4.0/). 